\section{LA CRISIS DE LA POLÍTICA CIENTÍFICA: PATOLOGÍAS DEGENERATIVAS Y TERAPIAS REGENERATIVAS. A MODO DE EPÍLOGO}

\author{
Emilio Muñoz \\ Instituto de Filosofía, Centro de Ciencias Humanas y Sociales, CSIC \\ $y$ Red CTI, CSIC
}

\begin{abstract}
The metaphoric assumption for science policy as its behaviour like an intelligent being has permitted to identify a so called "crisis" of this policy as the result of an aging process- more than sixty years have elapsed since the onset of the modern scientific policy. In that degenerative process, a series of pathologies had been diagnosed through a series of critical reviews undertaken by the author under a research programme on the "philosophy of science policy". By the use of medical and clinical metaphors, I have built a clinical record describing the pathologies and their diagnosis, referring them symbolically to mental and sensorial disorders. Among them, it can be mentioned amnesic process leading for instance to the dissociation between language (discourse) and actions; the poor understanding of the $R \& D+$ innovation concepts and their relationships; the mistaken use by problems of perception of the usual indicators; the transition from euphoria to depression (bipolar disorder) and the subsequent losses of the sense of reality, usually depending on external factors. In agreement with the analogies applied, therapies considered as the modern regenerative treatments are proposed to correct the "crisis" of the science policy. Among those therapies oriented to the care of degenerative processes, the incorporation (transplantation) of concepts such as those of "governance" and "spaces" into the sick body of the science policy is proposed. These old concepts are assumed again symbolically, to act as pluripotent, regenerative elements for a political management usually based on too simplified and traditional academic and bureaucratic approaches.
\end{abstract}

KEY WORDS: Medical metaphors, mental disorders, clinical record, governance, spaces.

\section{INTRODUCCIÓN}

En noviembre de 2007, el editor de este número de la revista, el doctor Manuel Fernández Esquinas, me propuso que contribuyera al mismo. A pesar de que estaba inmerso

\section{THE SCIENCE POLICY CRISIS: DEGENERATIVE PATHOLOGIES AND REGENERATIVE THERAPIES. AS MODE OF AN EPILOGUE}

RESUMEN: La aplicación de una analogía como ser vivo e inteligente a la política científica ha permitido identificar lo que he llamado "crisis" de esa política, como resultado de un proceso de envejecimiento -hace más de sesenta años que se inició la política científica moderna- en el que han intervenido una serie de patologías que han sido diagnosticadas a través de una serie de revisiones criticas emprendidas por el autor dentro del marco de un programa sobre "filosofía de la política científica". A través del recurso a metáforas médicas y clínicas he podido construir una historia clínica en la que se describen las patologías y sus diagnósticos, asociadas simbólicamente a trastornos mentales y sensoriales. Entre ellos cabe mencionar procesos de amnesia, del que es un ejemplo la disociación entre lenguaje y acción motora (discurso y acción); la deficiente comprensión de conceptos en el trinomio básico I+D+innovación y de las relaciones entre ellos; frecuentes errores de percepción (visión y audición) respecto al sentido y uso de los indicadores habituales; el tránsito, dependiente de circunstancias ajenas al propio objeto de la política de ciencia y tecnología, desde la euforia a la depresión (trastorno bipolar) y las consiguientes pérdidas de sentido de la realidad. De acuerdo con la aproximación metafórica aplicada, se proponen terapias para superar la "crisis" a las que se les aplica la analogía de las nuevas terapias regenerativas, orientadas a la corrección de procesos degenerativos. Entre ellas se incluye la incorporación (trasplante) en la marchita política científica de viejos conceptos como los de "gobernanza" y "espacios" que pueden actuar, de nuevo simbólicamente, como elementos troncales (pluripotentes) para que sirvan como regeneradores de una gestión política habitualmente apoyada en aproximaciones burocráticas y académicas esencialmente simples y tradicionales.

PALABRAS CLAVE: Metáforas médicas, trastornos mentales, historia clínica, gobernanza, espacios.

en una dinámica enloquecida de publicaciones, cursos y conferencias en los que tenía que reflexionar y discurrir acerca de las políticas científicas europea y española, así como de las relaciones entre estas políticas y los temas emergentes en la agenda política general como conoci- 
miento, desarrollo económico e innovación desde que se produjo el tránsito entre los siglos XX y XXI, decidí aceptar la invitación proponiendo un potencial artículo sobre el tema "La encrucijada de las políticas científicas: viejos principios y nuevos contextos".

La idea principal subyacente en este título era la pretensión de desarrollar el concepto de "crisis de la política científica" como fruto de un proceso de investigación alrededor de la filosofía de la política científica en el que he venido trabajando a lo largo de la última década.

Entre los factores que, en mi opinión, han contribuido a este diagnóstico de crisis y que ya anticipaba en el esquema del artículo que envié al editor, están los siguientes:

a) Las notables paradojas a las que se han enfrentado la ciencia y la tecnología en el nuevo contexto social y político de una globalización carente de otros objetivos que los de potenciar el individualismo y de confiar ciega, y casi únicamente, en la razón del mercado y de su valor principal que es el dinero.

b) Se reconoce que esta situación contextual casa mal con la naturaleza de la ciencia y de sus productos, los conocimientos científicos y técnicos que son un bien, considerado tradicionalmente como público, aunque de modo creciente se ha asociado con intereses privados por su contribución evidente al desarrollo económico y social, lo que conlleva la preocupación de la sociedad por estas dinámicas y la consiguiente implicación de la misma en la valoración, entendimiento y aceptación de los avances científicos y técnicos y,sobre todo de sus aplicaciones. De todo este cambio, surge la importancia de la ética y del derecho en relación con el progreso científico y el desarrollo tecnológico.

c) Todos estos procesos han generado a su vez una situación confusa en la que se han mezclado esfuerzos para encontrar modelos explicativos, que se han producido con una creciente intervención de los humanistas y científicos sociales para acercarse a una realidad, que muestra evidentes dificultades para su aplicación y explicación. Los diferentes modelos que han tratado de correlacionar la ciencia y la tecnología entre sí y de ambas con la producción de bienes y servicios a través de la innovación, han mostrado significativos déficit teóricos a pesar de revelar su utilidad para analizar las situaciones e incluso hasta comprender el sentido de los propios modelos. Esto ha conducido a una paradoja en el reconocimiento de los científicos sociales. Por un lado han experimentado frustraciones al no alcanzar el reconocimiento académico deseado dentro de su propia comunidad investigadora, mientras que sus modelos eran abrazados con fervor por los políticos y gestores en el ámbito de la ciencia y la tecnología. Sin embargo este abrazo ha sido similar al que realiza el oso, pues con esta declaración de fe, los políticos han puesto de relieve las dificultades para su aplicación, cayendo en evidentes incoherencias, que he resumido en la tradicional frase de "que una cosa es lo que se dice -los políticos y los gestores- -y otra es lo que se hace- y que sufren los cactores encargados de la ejecución". Ante estos fallos y conductas faltas de lógica, los científicos experimentales, a los que se les ha pedido que actúen en contextos diferentes a los que contribuyeron al desarrollo científico a partir de la segunda guerra mundial, han reaccionado, progresivamente de modo negativo,ante esta forma de pericia política, la de los "expertos en la elaboración de políticas" en la que excelen los científicos sociales, que no han buscado o no han conseguido la colaboración de los científicos cultivadores de las ciencias naturales ni incluso la de los ingenieros y tecnólogos, a pesar de que todos ellos en períodos de crisis -y hay testimonios sobre esta circunstancia en la crisis de 1990- la buscaron como alianza estratégica. Esta pérdida de oportunidad para lograr la conexión entre expertos de unos y otros campos científicos y académicos lo considero un error estratégico y científico de los cultivadores de las ciencias sociales y las humanidades como he denunciado en dos recientes textos: uno es el prólogo al libro Economía del conocimiento y la innovación. Nuevas aproximaciones a una relación compleja y el otro es un artículo en el número de noviembre de 2008 de la revista Temas para el Debate (Muñoz, 2008a y b).

Hoy, casi un año después, no me siento con fuerzas para escribir un nuevo artículo en el que volver a destilar reflexiones sobre las que, con mayor o menor claridad y coherencia, he venido insistiendo sobre estos puntos. Sin embargo, ante la insistencia del editor, el doctor Fernández Esquinas y en virtud del respeto profesional y la amistad 
personal que le profeso, me decido a escribir este artículo, a modo de epílogo y como punto y aparte a mis reflexiones, en el que habrá abundantes autocitas, por lo que ya pido perdón anticipadamente.

Para desarrollar el tema, transitaré desde la metáfora cartográfica que informaba el título anterior con referencia a la encrucijada, para entrar en las metáforas biomédicas como analogía explicativa de las patologias que sufren las políticas científicas y tecnológicas desde mi punto de vista. Por ello, el resto del artículo se construye a modo de historia clínica en relación a los factores diagnósticos que ya se han presentado en la parte introductoria.

\section{UNA hiStORIA CLÍNICA de LAS PATOLOGÍAS de las políticas CIENTífICAS}

\subsection{Trastornos mentales y neurodegenerativos}

Entre estos trastornos incluiría la amnesia, la disforia, la disociación entre lenguaje y acción motora conduciendo casi a situaciones de trastorno bipolar o a procesos esquizofrénicos.

A pesar de no ser un experto en economía y ser un casi total lego en sistemas financieros, no he andado muy desencaminado al asociar los problemas existentes en la agenda y la gestión política de la ciencia y la tecnología a un contexto anormal, como el que ha presidido nuestros recientes desarrollos económicos, alejado de la racionalidad y la responsabilidad que son propias del buen quehacer científico y tecnológico.

Si no el primero, soy uno de los primeros en reconocer la importancia del desarrollo científico y técnico para el progreso económico y social lo que debe reflejarse en su traducción, por medio de la innovación, sobre todo tecnológica aunque no descarte otros tipos, en productos y procesos.

Esta declaración se puede sustentar en el recuerdo a mi trayectoria como Vicepresidente del CSIC (1980-1982) y como Director General de Política Científica y Secretario General de la CAICYT (1982-1986). A este respecto, recomiendo la lectura de un libro editado por el CSIC en 2007, homenaje al autor de este artículo, bajo el título Tiempos de ciencia y de política, prestando especial atención a las contribuciones de José Antonio Martín Pereda, Regina Revilla y Elena Castro, Alejandro Nieto, Armando Albert, Jorge Barrero y mi propio texto. También me gustaría remitir al lector al preámbulo de la conocida como Ley de la Ciencia de 1986 y a todo el proceso que rodeó su elaboración (Muñoz y Ornia, 1986; Muñoz y García Arroyo, 2006). Me parece pertinente señalar que este recuerdo al preámbulo de esa ley me ha venido durante la celebración de unas jornadas de Debate sobre el nuevo proyecto de ley de ciencia y tecnología organizadas por el Ministerio de Ciencia e Innovación los días 9 y 10 de octubre de 2008, tras la mención al mismo que hizo el diputado del PNV, José Ramón Beloki, como prueba de su vigencia.

A partir de estas referencias personales, se puede construir la plataforma desde la que elaborar el discurso que permite establecer los sintomas relacionados con las patologías enunciadas en el inicio de esta sección.

\section{- Política científica en Europa}

En una serie de cursos en los que he intervenido en los Máster sobre Estudios Sociales de la Ciencia en las Universidades de Salamanca y Oviedo, desarrollando la cuestión de los orígenes de la política científica y tecnológica en Europa, he llegado a una panoplia de conclusiones con puntos que soportan la atribución de patologías de olvido de memorias a los actores en la gestión de política de ciencia y tecnología a lo largo de las últimas décadas.

Extraigo algunos párrafos de textos desarrollados en esos cursos.

"En contraste con la opinión normalmente sustentada por los analistas en política científica de que la política científica tuvo su origen en la propuesta del ingeniero norteamericano Vannevar Bush con su documento Science, The Endless Frontier, hay que señalar que Europa tuvo su propia dinámica en los períodos de postguerras mundiales y puso en marcha iniciativas y estrategias de cierto calado.

Europa tenía que asumir que en los años de la posguerra mundial llevaba 150 años de civilización científica -son hoy más de doscientos- por lo que la política científica era

ARBOR CLXXXV 738 julio-agosto [2009] 837-850 ISSN: 0210-1963 
un objetivo claro en las sociedades europeas con el fin de promover el progreso científico.

Esta estrategia debía encontrar su correlato con un discurso que implicara claramente a los gobiernos para movilizar a las sociedades y configurar opiniones públicas favorables al esfuerzo público que era necesario acometer. Con ello se pretendía conseguir al mismo tiempo la positiva intervención del sector privado. Es importante destacar la coincidencia en las estrategias con las que se vienen defendiendo y poniendo en práctica en las dos últimas décadas y con particular vigencia en estos tiempos.

De este breve recorrido puede sacarse ya una importante conclusión que va a presidir este análisis introductorio de la política científica y tecnológica en Europa. La conclusión es la siguiente. 'Las bases y grandes principios de la política científica en las sociedades occidentales fueron establecidas en los primeros años de la segunda posguerra mundial.' En este proceso hubo una coincidencia temporal entre Europa y los Estados Unidos, aunque fueron éstos con la filosofía de la 'La ciencia, frontera sin límites' y su poder hegemónico mundial los que aparecen retrospectivamente como los precursores y líderes de la iniciativa.

Desde los primeros momentos, la política científica en Europa contempló la necesidad de que existieran relaciones entre el mundo científico, el entorno privado como motor empresarial y productivo, y el ámbito político como promotor y gestor. De ahi que fuera necesario pensar y debatir sobre las posiciones que en estas relaciones debian contemplar los distintos actores, posiciones que respondieran a dinámicas variables, aunque ajustándose a los principios de separación (independencia) y responsabilidad (auto y exoexigencias).

En todo este proceso se estableció la conveniencia de explorar (ya en aquellos momentos) las relaciones entre ciencia y sociedad o entre científicos y gobierno.

Adquiere carta de naturaleza, en el ámbito/espacio de la producción de los conocimientos, la división entre ciencia básica cuyo objetivo es conocer -contribuir al aumento del acervo de los sabores y que forma parte de la vida académica-, ciencia aplicada cuyo fin es actuar y formar parte esencialmente del mundo más técnico y emprendedor, y la tecnología, que unas veces es motor para la producción de los conocimientos y otras veces es un resultado de los desarrollos científicos, como proceso híbrido e interactivo que da cuenta de la dinámica de la ingeniería y de la dinámica de los centros politécnicos.

Esta división que procede más de la escuela europea que de la norteamericana, ha tenido efectos positivos para la estructuración y organización de los mecanismos de promoción y fomento de la investigación y de las infraestructuras, pero ha traido asimismo problemas por su tendencia a la separación y a la compartimentalización, cuando se ha hecho preciso conectar e integrar a los agentes productores del conocimiento y a sus usuarios, es decir la academia y la empresa.

Es interesante, sin embargo, subrayar que Europa ya se plantea en aquellos momentos de mitad de siglo XX, la reflexión acerca de si la ciencia debe ser considerada como una función social, como un bien público o colectivo, a la par que se reconoce la actividad científica como profesión.

En general, cabe concluir que en Europa, con una sorprendente convergencia entre democracias liberales y paises socialistas, unas democracias europeas que en muchos casos se encuentran a medio camino entre el liberalismo y el socialismo, apuestan por la ciencia como elementos básicos para el desarrollo económico o social -no apostar por el desarrollo científico y técnico supone ir hacia el estancamiento. La ciencia se convierte en elemento básico del discurso político y la elaboración de estrategias y planes científicos y tecnológicos es un punto común del ejercicio político."

\section{- Política cientifica y tecnológica en España}

Más sorprendente aún a este respecto es el caso español a pesar de las limitaciones que han caracterizado sus políticas de ciencia y tecnología a lo largo de su historia.

En un artículo (Muñoz y Sebastián, 2007), incluido en el libro Cien años de Política Científica en España, editado por Ana Romero y María Jesús Santesmases, se han explorado los orígenes de la política científica española, atendiendo para su caracterización al papel circunstancial ejercido por la ciencia en su configuración y desarrollo. De ese artículo, se pueden extraer unos párrafos que ofrecen detalles argumentativos para el propósito que nos ocupa y que se ofrecen a continuación. 
"El origen de la potencial política científica española se sitúa en la primera década del siglo pasado, con la creación, en 1907, de la JAE como organización básica para llevar a cabo una política para la ciencia en la que la línea de actuación de fomento debe concluir a la formación de recursos humanos y a la producción de conocimientos científicos de alcance.

En la JAE se crean infraestructuras en forma de institutos y laboratorios para la ejecución de estos objetivos. La línea de actuación se diversifica, en un primer salto de búsqueda, de la opción de una política por la ciencia. Con el objetivo de contribuir al desarrollo pesquero y marítimo se crea, en 1914, el Instituto Español de Oceanografía (IEO).

La política por la ciencia encuentra un nuevo apoyo en el ámbito de la productividad industrial con la publicación, en 1929, de un texto refundido de la Ley y de la Propiedad Industrial.

Tras el doloroso paréntesis de la Guerra Civil, todos estos esfuerzos son escondidos y subsumidos en primera instancia con la creación, en 1939, del Consejo Superior de Investigaciones Científicas (CSIC) que trataba de abarcar las dos lógicas de 'política para y por la ciencia'.

Perseguia la conexión entre investigación y centros de ciencia aplicada para el cultivo de la técnica con el fin de aprovechar, en beneficio de la riqueza y prosperidad del país, todas las energías fisicas y biológicas de nuestro territorio. En 1940 se regulaba el funcionamiento del organismo. En un régimen donde no parecía existir una política de ciencia y tecnología, el CSIC, a pesar de la influencia de su fundador e inspirador José María Albareda, no tuvo una tarea fácil ni una trayectoria bien definida en el contexto político.

En paralelo, empezaron a surgir iniciativas sectoriales, la mayoría de las cuales se podian englobar bajo la lógica de políticas por la ciencia que entraban en colisión con los objetivos y las pretendidas acciones del CSIC.

Dentro de la época tecnocrática del régimen franquista se produce un acontecimiento de la mayor relevancia para los primeros asentamientos de la política científica española: la creación de la Comisión Asesora de Investigación Científica y Técnica (CAICYT) por un Decreto, de febrero de 1958, que se convalida como Ley en diciembre de dicho año. Constituye un instrumento para llevar a cabo una política por la ciencia con el objetivo de situar en un marco relevante, dentro de la acción política, las cuestiones relativas a la investigación y buscar una cierta homologación con el plano internacional.

Es importante notar que, desde la creación del CSIC en 1939, trascurren 20 años en que se genera legislación sobre centros de ejecución de investigación básica y aplicada, pero sin ninguna actuación encaminada a desarrollar normas relacionadas con los niveles de planificación y financiación. La creación de la CAICYT marca un punto de inflexión a partir del cual se empieza a situar la investigación científica y técnica, con la llegada de los tecnócratas, en el foco de la acción política. A partir de ahí entra en acción la Comisión Delegada del Gobierno y se crean alguna Comisión y diversos organismos.

La creación del Fondo Nacional para el desarrollo de la Investigación Científica y Técnica (FONDICYT), marca otra actuación acorde con la lógica de la política para la ciencia, ya que se establece un instrumento de fomento, no sólo para el mundo de la ciencia sino también para el entorno empresarial, con el fin de promover la investigación aplicada, el desarrollo tecnológico e, incluso, los procesos de innovación tecnológica. Es importante señalar como dato preocupante para retener, que este Fondo Nacional se crea ocho años después de la creación de la CAICYT. Este dato hace dudar de la voluntad real de considerar la ciencia y la tecnología como prioridad política. Como un instrumento dirigido al entorno empresarial se establecen los Planes Concertados de Investigación con cargo al Fondo Nacional".

Estas consideraciones sobre el caso español desde el punto de vista de la protohistoria de su política científica, sirven como argumento para reforzar el punto de vista de la existencia de la amnesia como patología recurrente en los diseños de la política científica, española en este caso. Poco nuevo hay en el discurso, lo que falla es la acción y la realidad social y política.

\section{- Distancia entre discurso y acción. El espacio europeo}

En esta línea de argumentar acerca de la existencia de evidentes distancias entre discurso y acción en el ámbito de la ciencia y la tecnología, se puede hacer mención a los 
esfuerzos europeos para conseguir el cumplimiento de la agenda de Lisboa 2000 (Muñoz et al., 2005).

Una primera y flagrante contradicción que se recoge en el prefacio de ese documento (Muñoz et al., 2005), se sitúa en los mismos procesos de la Comisión Europea para asentar la declaración de "convertir a Europa en el año 2010 en la economía más competitiva del mundo basada en el conocimiento... y para ello se pretendía alcanzar el $3 \%$ (del producto interior bruto) de inversión en investigación y desarrollo". En efecto, en la "Comunicación de la Comisión al Parlamento Europeo, al Consejo Económico y Social y al Comité de las Regiones. Hacia un espacio europeo de investigación", COM (2000) 06, Bruselas 18/01/2000, la primera preparatoria en relación con la propuesta de Lisboa, la iniciativa europea se asentaba sobre el concepto de espacio europeo de investigación, y en ella se entremezclaban los conceptos de conocimiento, investigación y tecnología, pero no se hacía mención a la innovación. Por otro lado, la referencia a la dimensión geográfica a la que se aludía en esa Comunicación era la nacional.

Sin embargo, casi dos años después, en el último trimestre de 2001 aparecería una Comunicación de la Comisión con aparente menor alcance ya que no tenía ningún destinatario político representativo específico [Comunicación de la Comisión "La dimensión regional del Espacio Europeo de investigación", COM (2001) 549 final, 03/10/2001]. En esta Comunicación reaparece la conexión entre investigación e innovación, en línea con las tradicionales preocupaciones europeas, dimensiones que se asocian además con otros niveles geográficos y políticos: el plano regional, los fondos estructurales, las diferencias regionales y el marco de ampliación. Una comparación entre las dos Comunicaciones pone de relieve contradicciones en las políticas europeas en ciencia y tecnología, en investigación e innovación. En lo que aparece como una estrategia de adaptación al marco de la agenda de Lisboa 2000, a lo largo de 2002 y 2003 , el marco normativo de la ciencia y la tecnología europeas se ha visto inundado por una serie de Comunicaciones, algunas de ellas emitidas con ambición política de alcance al haber sido dirigidas al Consejo, al Parlamento Europeo, al Comité Económico y Social y al Comité de las Regiones.

Todas estas iniciativas ponen de manifiesto en nuestra opinión, la existencia de un ambiente confuso, aunque sin duda reflejo de la importancia que se le presta a la investigación, al desarrollo tecnológico y a la innovación como pilares fundamentales para el desarrollo económico, social y político de Europa.

En ese prefacio, ya citado, se apuntaba que todas estas contradicciones entre las apuestas por la ciencia y la tecnología -espacio europeo de investigación y las preocupaciones por articular sus avances con la innovación- nos movian a adoptar posiciones de escepticismo acerca de alcanzar los objetivos máximos de las políticas de I+D+i en el seno de la Unión Europea. La desconfianza surge, decíamos, "por las distancias que se detectan entre el discurso, la estrategia, los intereses y las acciones (implementación). Se percibe, además, dentro de esa línea de divergencias, que continúa el predominio de la visión lineal en el diseño de los objetivos y en la práctica de la promoción de la ciencia y la tecnología, y la innovación en Europa, aunque se mezcle con aproximaciones sistémicas e interactivas".

En ese trabajo previo (Muñoz et al., 2005) realizado para la Academia Europea de Ciencias y Artes (AECYA, España) se cuestionaba la idea de un Espacio Común del Conocimiento en Europa, particularmente difícil de contemplar desde la perspectiva española. Al analizar los tres lados del triángulo que componen la educación, la investigación y el desarrollo tecnológico, y estos dos elementos en su relación con la innovación en la nueva orientación de la disección europea del polinomio $1+D+i$, se pudo detectar la existencia de importantes diferencias entre los actores y sus comunidades, entre los procesos de formación, evaluación, responsabilidad y ética (normas y valores), así como en los de la intervención social (cultura, percepción, difusión, comunicación y participación).

De ahí que una de las más importantes conclusiones de ese trabajo fuera que:

"Un análisis retrospectivo acerca de lo que hay detrás del movimiento de Lisboa 2000 y de los sucesivos cambios que tratan de proponerse para un mejor desarrollo científico y tecnológico con posible (y deseable) incidencia en la innovación, revela la existencia de ambigüedades, confusión, descontento, inadecuación de los modelos y mezcla de diferentes modelos en la implantación de las estrategias y planes, diferencias notables entre lo que se propone (o dice) y lo que se hace" (Muñoz et al., 2005, Muñoz, 2007). 


\subsection{Trastornos sensoriales y neuromotores}

Entre estos trastornos incluiría problemas en la percepción derivados de dificultades en la visión (ametropia), en la audición (hipoacusia) y el olfato lo que se traduce en alteraciones en la comprensión de las relaciones entre las políticas científicas y tecnológicas, los desarrollos económicos y sociales y la repercusión que sobre estos factores tiene la innovación, así como en errores de medida para el diseño de estrategias y políticas.

En el prólogo del libro Economía del conocimiento y de la innovación. Nuevas aproximaciones a una relación compleja (Cañibano, Encinar y Muñoz, 2008), he planteado algunas reflexiones sobre estos temas, entre las que transcribo algunas a continuación (Muñoz, 2008a).

"Un primer dato a constatar es que nos encontramos en una situación paradójica ante los desarrollos científicos y tecnológicos. Nunca hasta ahora han contado la ciencia y la tecnología con mayor reconocimiento (¿es sólo aparente?) en las agendas de los paises desarrollados, pero tampoco nunca se habian presentado mayores problemas para aplicar la racionalidad científico-técnica a la solución de los problemas y desafíos a los que se enfrenta la comunidad internacional en lo que respecta a la sostenibilidad de su desarrollo económico y social, sobre todo si atendemos a las complejas reacciones sociales que se suscitan en el litigio entre necesidades y demandas de los ciudadanos y sus temores respecto a las acciones cientificas y tecnológicas, temores pocas veces sustentados en un armazón científico y técnico sólido serio, sino esencialmente inspirados por creencias y conflictos de intereses.

Dos son, en mi opinión, los principales factores de entorno que condicionan esta difusa situación: el tránsito entre dos milenios con el imaginario que eso arrastra, y la globalización que los acompaña, lo que ha obligado a plantearse nuevas preguntas acerca del futuro y a buscar nuevos objetivos que den respuesta a las mismas.

Desde las Cumbres de los países más ricos (el célebre G8) hasta la Unión Europea, pasando por las organizaciones internacionales encargadas de reducir la brecha entre el desarrollo y subdesarrollo, las agendas han ido incorporando un número creciente de preocupaciones y referencias sobre las relaciones entre conocimiento científico y técnico y rendimiento económico ('performance').

Los conceptos que han ido aflorando como destilado de esos procesos son: economía y crecimiento sostenido; construc- ción de un sistema de comercio apoyado en procedimientos basados en ciencia sólida y reglas juiciosas; políticas de empleo sustentadas en más y mejor educación; información y buen gobierno en la gestión de la estabilidad social; la profundización en la cooperación para el desarrollo; la protección del medio ambiente; la necesidad de garantizar la confianza pública para gestionar tanto las estrategias de la defensa como la protección de bienes colectivos.

Las políticas de fomento de I+D experimentaron un notable giro a partir de la década de 1970, como consecuencia de la primera crisis energética experimentada por el modelo occidental de desarrollo basado en el impulso norteamericano que siguió a la Segunda Guerra Mundial.

Es en este periodo cuando surge la moderna política científica, que fue construida alrededor de los principios de 'ciencia como frontera sin límites' (corresponde a lo que en el texto que nos ocupa -el libro Economía del conocimiento y la innovación. Nuevas aproximaciones... se refiere como 'ciencia abierta'), política científica que algunos hemos identificado como 'política para la ciencia' (Muñoz y Sebastián, 2007). Los momentos de profundo cambio se reflejaron en diversas dinámicas, entre las que cabe mencionar el cuestionamiento de los modelos interpretativos de la relación entre los factores del bionomio I+D entre sí y de ellos con la innovación; la búsqueda de las posibles ventajas del modelo tecnoeconómico japonés frente a los modelos occidentales; la atención prestada a la organización de las actividades investigadoras e innovadoras de las empresas -es decir el análisis de sus capacidades creativas- para contribuir a su desarrollo económico intrínseco y con ello para incidir en el del sector en el que operan; así como en el entorno geográfico en el que asentaron sus actividades.

Es en este contexto donde tiene lugar la emergencia de las políticas europeas en ciencia y tecnología. Estas políticas de I+D en Europa han ido alcanzando creciente importancia desde finales de la década de los ochenta del siglo pasado, aunque también estas políticas, articuladas alrededor de los Programas Marco, han experimentado cambios significativos por lo que la continuidad y la fidelidad a los objetivos no son, precisamente, las características predominantes de esta actividad europea...

La situación ambivalente de la I+D comunitaria en la que se combinan aspectos positivos y negativos -todos ellos debatidos frecuentemente en relación al discutido concepto de 'paradoja europea'- ha conducido a la búsqueda de nuevas estrategias entre las que destaca, como andamiaje sobre el que se construye el discurso europeo, la declaración del Consejo Europeo de Lisboa (marzo del año 2000), en la 
que se proponía 'hacer de Europa la economía basada en el conocimiento más competitiva y dinámica del mundo' a finales de la década, es decir en 2010.

Esta estrategia suponía la asunción de nuevos ejercicios como la recopilación periódica de datos sobre indicadores, la elaboración de líneas directrices para las políticas nacionales y el aprendizaje mutuo -por medio del proceso denominado 'coordinación abierta'- iniciativas que debían contar además con el apoyo de los expertos (proceso de revisión y consulta con los pares).

Esta orientación no se ha llevado a cabo de modo homogéneo, poniendo de manifiesto la existencia de nuevo de una situación paradójica. Mientras que el foco de la política europea en cuestiones científicas y tecnológicas se situaba en el año 2000 en el terreno de la investigación (producción de conocimiento), los esfuerzos para desarrollar los trabajos necesarios para la implementación de la estrategia de Lisboa se han concentrado en la innovación, que aunque considerada como un campo, no reflejaba su centralidad en los Programas Marcos V y VI, ya que estos Programas se han concentrado en los dos primeros elementos del trinomio I+D+i, es decir en la I+D.

Esta situación, evidentemente contradictoria, se abordó con iniciativas paralelas, como fue el caso del Esquema Orientativo sobre la Innovación que se puso en práctica como proyecto piloto en 1999, y fue lanzado oficialmente en el año 2000 con objetivo de acelerar la adopción de políticas de innovación y de optimizar los resultados de la innovación.

De nuevo, los resultados de esta acción parecen limitados. Quizá el elemento más visible de las actividades del Esquema Orientativo es la elaboración del Cuadro europeo de indicadores, construido con una visión sistemática, en la que se incorporan 20 indicadores (de innovación) que abarcan los recursos humanos, la creación de nuevos conocimientos, la difusión y aplicación de conocimientos, la financiación de la innovación, la evolución de los productos y mercados con especial atención al sector servicios. Este cuadro de indicadores se complementa con otros indicadores, promovidos por la Dirección General de Empresa (DGE), la institución que en la Comisión Europea asume la responsabilidad de promover la innovación en la actualidad, y que por cierto actúa con escasa interacción con la Dirección General de Investigación, responsable a su vez de la gestión del Programa Marco. Esta situación de disociación funcional de las dos Direcciones Generales, limita las posibilidades de ejercer acciones de fomento directo sobre la innovación con lo que es extremadamente difícil reconocer, identificar y evaluar sus políticas, si es que existen. Entre estos otros indicadores se incluyen los datos sobre empresas y el informe anual sobre competitividad, asi como los datos que se derivan del análisis de la dinámica innovadora en sectores con diferentes niveles de tecnología: baja, media-baja, media-alta y alta. Los datos recopilados a nivel de países, muestran que los países con buena actuación en los sectores de alta tecnología también presentan datos favorables en el resto de los sectores con niveles inferiores de tecnología. Los sectores dinámicos de alta tecnología parecen ejercer un efecto motor sobre el resto de los sectores económicos, mientras que lo contrario no ocurre. Aquellos países basados en sectores de limitados niveles tecnológicos no consiguen poner en marcha sectores de alta tecnología ni de influir decisivamente en su desarrollo.

A partir de los argumentos desgranados es difícil concluir que exista una relación directa entre políticas científicas y tecnológicas, orientadas a fomentar la producción de conocimiento científico y técnico, y el desarrollo de innovaciones -dejando aparte las tecnológicas-, que contribuyen al crecimiento económico y al bienestar social ejemplificados en la creación de empleo y en la mejora de las condiciones de vida y trabajo, procurando además mantener las mejores condiciones del medio en el que se desarrollan las actividades productivas y en el que transcurren las vidas de los usuarios de los productos resultantes de tales actividades.

Ya he señalado en algunas ocasiones que las políticas científicas y tecnológicas,destinadas a fomentar la producción de conocimiento científico y técnico, siguen operando con instrumentos y evaluándose con indicadores de clara inspiración en el modelo lineal, independientemente que estas políticas se diseñen y gestionen a nivel supranacional, nacional y local. En virtud de esta asunción es por lo que, dentro de un programa sobre 'filosofía de la politica científica' he venido denunciando las contradicciones existentes en estas políticas, la distancia entre el discurso y las acciones (Muñoz y cols., 2005; Muñoz, 2006, 2007), lo que dificulta en mi opinión tanto la interpretación como la puesta en práctica de la estrategia de Lisboa 2000".

\section{Propuestas de terapias. Las terapias REGENERATIVAS SOBRE LA BASE DE VIEJOS CONCEPTOS}

De acuerdo con la aproximación metafórica escogida para analizar la crisis de la política científica después de 70 
años de práctica, las analogías biomédicas y clínicas empleadas llevan a la asunción de que la citada política es como un organismo vivo, que ha llegado a la senectud tras sufrir una serie de patologías, esencialmente neuronales por tratarse de un ser vivo dotado de inteligencia, a lo largo de su vida.

Consecuentemente, la propuesta de terapias acude a la analogía con las terapias más actuales y que ofrecen más expectativas para la corrección de procesos degenerativos. Me refiero a las Ilamadas "terapias regenerativas" en las que se persigue el potencial de transformación de elementos celulares básicos, las células madre totipotentes o pluripotentes según los orígenes, para su implante en el organismo enfermo en alguno de sus órganos o tejidos.

En este caso, se recurre a la utilización de elementos conceptuales básicos como de los "gobernanza" y "espacios" para su implantación en las políticas de ciencia y tecnología con objetivo de conseguir una mejora en sus puntos críticos.

De nuevo acudo a trabajos personales previos para desarrollar estas propuestas extrayendo argumentos ya publicados.

\subsection{Gobernanza y ciencia y tecnología (Muñoz, 2005)}

"La implantación del término gobernanza en el ámbito de la ciencia y la tecnología ha llegado de la mano de la dinámica política de la Unión Europea que ha caracterizado los pasos finales del desarrollo del $V$ Programa Marco y el diseño y puesta en marcha del VI Programa Marco. Un conjunto de iniciativas sociopoliticas(comunicaciones, decisiones, identificación y selección de prioridades) promovidas por la Comisión Europea en relación con la reflexión relacionada con la concepción del Espacio Común Europeo en I+D(ERA) han ido configurando un paisaje en el que el concepto gobernanza ha alcanzado carta de naturaleza. A partir de ahi se ha producido una floración de publicaciones en que se relaciona la dinámica de la promoción de la I+D, su financiación con la intervención de diferentes formas de agencia y la gestión de los recursos humanos y materiales con dicho concepto de gobernanza. Sin embargo, en esas publicaciones parece que se recurre al uso del término gobernanza de forma casi automática, seguidista de las iniciativas promovidas por la Comisión Europea, pero sin aporte analítico ni sustrato descriptivo.

Parece evidente que la misión fundamental que atribuye la propuesta de la Comisión al término gobernanza es su operatividad para afrontar el problema de los diferentes niveles de promoción y gestión de la ciencia y la tecnología, y de su instrumento político -la política científica-tecnológica y la innovación- en un contexto como el europeo donde coexisten lo supranacional, lo nacional, lo regional e, incluso en ciertos lugares o momentos, lo local. De ahí que la línea elegida por los decisores en política científica y tecnológica, y seguida por los estudiosos del tema, sea la que trata de comprender el papel de los 'espacios' y la influencia en ellos de la existencia de varios niveles políticos - propuesta, decisión y ejecución- y de la participación de diversos actores. En resumen, el reto de la 'coordinación operativa' ante la división del trabajo en 'I+d e innovación'.

Esta aproximación es interesante, pero parece también, en una primera mirada, limitada en sus fundamentos y en sus aspiraciones. Se presenta asimismo arriesgada, pues responde a un seguidismo mimético por parte de los analistas, con lo que nos podemos ver envueltos en círculo vicioso: se estudia aquello que los decisores políticos han introducido, mientras que se renuncia a entender lo que sustenta la oportunidad, validez y el potencial del concepto para desarrollar una dinámica racional en términos sociales, económicos y políticos en el ámbito de la ciencia y la tecnología.

No podemos olvidar los contextos sociales de preocupación y contestación en que se mueve la idea de 'progreso' y la aplicación de las nuevas tecnologías en las sociedades avanzadas per se y en su relación con el mundo en desarrollo, menos avanzado. Temas como riesgo, incertidumbre, responsabilidad, participación social, democratización de la ciencia (¿y la tecnología?), intervención y 'laissez-faire', relación entre lo publico y lo privado, patentes y subdesarrollo, están muy presente en la conflictiva agenda socio-política que marca el período comprendido entre el final del siglo $\mathrm{XX}$ y los inicios del siglo XXI".

A la vista de estas dificultades, hemos trabajado en los años 2004-2006 en un proyecto sobre el concepto de "gobernanza y sociedad civil en ciencia y tecnología en España", encargado por la Fundación Española para la Ciencia y la Tecnología (FECYT) al Instituto de Filosofía del CSIC.

Fruto de ese trabajo ha sido la propuesta de una definición de gobernanza en los términos que se transcriben a continuación: "La creación de valor público ha sido utilizada como argumento para proponer la necesidad de gobierno 
estratégico en el sector publico. Este gobierno estratégico encuentra su proyección integradora en el concepto de gobernanza. De acuerdo con todo lo expuesto, se puede concluir que gobernanza, en una definición ampliada de la que recoge el Diccionario de La Lengua, es la puesta en práctica de formas de gobierno estratégicas para poner de relieve el valor de lo público a través de la relación entre sociedad, mercado y Estado y conseguir de este modo un desarrollo socialmente sostenible. Una serie de técnicas, con un creciente de nivel de participación social y que deben estar en proceso de continua revisión y mejora, emergen como puntos de apoyo significativos para la consecución de estos objetivos.

La definición de 'gobernanza' que acabamos de delinear lleva aparejada una clara opción sistémica, aunque esta opción encierra una mayor complejidad que la simple concepción de sistema como conjunto de elementos afines que cooperan. Se concibe como un sistema político, desarrollado por la escuela norteamericana de ciencia política, en el que se gobierna con la intervención de una variedad de actores y que requiere una arquitectura para la decisión en varios niveles.

En el ámbito de la ciencia y la tecnología, la visión predominante, desde mediados de los años 50 hasta la década de los setenta en esa misma centuria, ha sido el modelo lineal que conectaba ciencia, investigación, desarrollo e innovación en una cadena, en la que cada uno de los eslabones tenía su propia dinámica y actuaba de acuerdo con patrones especificos, mientras que la influencia sobre el siguiente eslabón se limitaba a un flujo de materiales (conocimiento en términos generales).

Las dificultades experimentadas por el modelo lineal para explicar la influencia de la actividad científico-técnica (tecnocientífica en la nomenclatura de J. Echeverría, 2002, 2003) en la producción tecnológica y en la producción de innovaciones condujo al concepto de 'sistema de innovación' como modelo interpretativo de tales procesos, promovido por la teoría económica de carácter evolucionista y neoinstitucionalista (Smits y Kuhlmann, 2004).

El concepto de 'sistema de innovación' trata de integrar la práctica totalidad de los factores que intervienen en la producción y utilización de ciencia y tecnología para conseguir su transformación en bienestar económico y social. Sin embargo, este concepto, entendido como conjunto de elementos por su naturaleza y estructura, presenta problemas para el caso de la ciencia y la tecnología. En este conjunto existen elementos que pueden y deben cooperar, pero cuya afinidad es cuando menos limitada. De hecho, el concepto está centrado, como su propio nombre indica, en el proceso de innovación y se focaliza en los intereses y estrategias empresariales que en esencia son diferentes de los que movilizan los otros dos elementos del trinomio I+D+i, la investigación y el desarrollo tecnológico. En la definición propuesta por Metcalfe (1995) un 'sistema de innovación' abarca todas las instituciones implicadas en la investigación científica y su desarrollo; instituciones que son responsables de la acumulación y difusión del conocimiento, proceso que encuentra su reflejo en la educación y formación de la ciudadanía con potencial impacto en las condiciones de empleo y trabajo, incide en el desarrollo tecnológico, para por último influir en la producción de nuevos productos y procesos así como en su distribución. Esta concepción de 'sistema de innovación' atribuye al conocimiento el papel fundamental, lo considera soporte y armazón para el eficaz funcionamiento de ese sistema.

La atribución de este papel central al conocimiento 'experto' sirve para poner de manifiesto que no todas las instituciones tienen similar importancia en la configuración y puesta en acción de un 'sistema de innovación'. La relevancia de la comunidad 'tecnocientífica' en la 'gobernanza' de la ciencia y la tecnología es evidente, con lo que confiere a la 'gobernanza' de este ámbito sociopolítico un marcado carácter diferencial con respecto a otros tipos de 'gobernanza'.

Desde hace siglos, los científicos avanzaron en el diseño y puesta en práctica de las instituciones por las que vehicular la difusión de su actividad y establecer plataformas para sus críticas y discusión.

Sin embargo, la evolución experimentada por este tipo de procesos de gobierno ha sido influenciada por el cambio en los contextos de desarrollo científico y tecnológico (Muñoz 2002). La conexión e interacción entre los conceptos de 'sistema político' y 'sistema de innovación' ha determinado la heurística para analizar el sistema de 'gobernanza' en relación la ciencia y la tecnología. La revisión crítica del modelo lineal ha servido para identificar cuatro brechas o desfases entre la linealidad y su repercusión en un entorno complejo. Esas brechas se dan entre:

- La práctica política y el mundo de la ciencia y la tecnología,

- Las disciplinas científicas,

- Los actores y gestores políticos y los ciudadanos,

- Los expertos y los legos,

y conducen a una nueva aproximación sistemática, pero que comprende dos nuevos conceptos: uno de carácter estructural (espacio) y otro de dimensión funcional (gobernanza). 
Dentro de esta visión sistemática, orientada preferentemente a comprender el modo en que las organizaciones generan innovaciones, la actividad científica ha tenido que combinar sus formas habituales de gobierno en las que sus actores, según se ha visto, ejercian funciones polivalentes como actores, gestores, políticos y usuarios, con otros modos encaminados a aumentar el número de funciones para conseguir una mayor conexión con la sociedad por medio de la asunción de crecientes cotas de responsabilidad y de intervención en los procesos de difusión y divulgación del conocimiento. El giro en el modo de producción de avances científicos y técnicos, cada vez más conectado con el mundo empresarial, ha colocado a los expertos 'tecnocientíficos' ante un dilema: por un lado, ha aumentado su valor $y$ participación en el área de la asesoría relacionada con la empresa: licencias, patentes, interacción a distintos niveles a empresas ('spin-offs', 'start-ups'), pero, por otro lado, se ha incrementado la desconfianza hacia su independencia por parte de los sectores de la ciudadanía más críticos sobre la avenida del progreso y sus consecuencias.

En un orden parecido de cosas, se sitúa la intervención de la comunidad experta en problemas científico-tecnológicos de carácter público. Inmersos en una sociedad del riesgo, las actitudes del público ante nuevos productos o procesos tecnológicos son muy variadas y complejas, a cuyos retos de regulación y valoración deben tratar de enfrentarse los expertos, bien a través de agencias especificas o a titulo individual para ejercer el asesoramiento en instituciones de naturaleza generalista. En todos estos temas, la comunidad experta debe afrontar la búsqueda de confianza, junto a la necesidad de aportar conocimiento cualificado para una gestión estratégica de problemas de base científico-técnica y con incidencia en la agenda política como la seguridad, la política de I+D en diferentes niveles, la introducción de nuevas tecnologías o la repercusión socioeconómica".

\subsection{Espacios de conocimiento, gestión y gobernanza} (Muñoz, 2007)

Del artículo publicado en la Revista Iberoamericana de Ciencia, Tecnología y Sociedad (Muñoz, 2007) en el que he desarrollado una reflexión, desde el punto de vista español, sobre la oportunidad de aplicar conceptos como el de sociedad de conocimiento junto con los de "espacios" y "gobernanza" para dar cuenta de la dinámica y las problemáticas presentes en la política y la gestión de la ciencia y la tecnología, extraigo unos puntos para consideración.
"Dentro del contexto de un programa sobre 'filosofía de la política científica', merece especial atención en nuestros días seguir la evolución de las orientaciones de la Unión Europea sobre políticas de ciencia y tecnología y su eventual incidencia económica y social, marcadas, en el tránsito intersecular, por la agenda de Lisboa 2000 en el plan político y por el diseño, gestión y desarrollo de los Programas Marco VI y VII en el plano organizativo e institucional. En estas nuevas orientaciones que revelan la necesidad de cambios importantes ante las limitaciones de los modelos aplicados para sustentar discursos anteriores, tropiezan desde los primeros análisis con barreras culturales y patrones de conducta que cuestionan la homogeneidad (¿universalidad?) de los planteamientos y la factibilidad de sus aplicaciones. Esta situación de conflicto ha llevado a la introducción de nuevos conceptos e instrumentos, como los de 'espacio' y 'gobernanza' en relación con la dinámica científica, tecnológica, que van a ser objeto central de este trabajo de reflexión.

A diferencia de lo que ocurre con el término 'knowledge', el concepto conocimiento en castellano no es unívoco para su inmediata traducción en que una sociedad basada en el desarrollo científico y técnico sea una 'sociedad del conocimiento.' Hay que cualificar esta sociedad como 'sociedad del conocimiento científico, tecnológico o tecnocientífico' so pena de caer en arriesgadas y peligrosamente aprovechadas imprecisiones. En efecto, se detectan importantes diferencias culturales respecto a lo que se entiende por conocimiento en castellano $y$, por lo tanto, al uso que se haga o se pueda hacer de este concepto en su asociación con el desarrollo económico y social.

Es particularmente interesante señalar las dificultades existentes para ajustar el concepto de conocimiento a otros que están presentes en el discurso político y social y que revelan la existencia de profundo cambio en el marco de una visión evolutiva que discurre por el tránsito entre sociedades y entre siglos (como es el caso de nuestra situación actual). Estos cambios con: 1) Geoestratégicos: plano macro (globalización); planos meso y micro (servicios); 2) Culturales: recursos humanos (información); nuevas tecnologías (aprendizaje): 3) Científico-técnicos: producción de conocimiento (modo 2, tecnociencia; aceptación social -percepción-; regulación).

Parece lógico proponer una estrategia analítica en la que se pueda profundizar en la forma en que se producen y gestionan los diferentes tipos de conocimiento recurriendo al concepto de espacios donde los actores se reúnen y actúan de acuerdo con sus dinámicas por medio de procesos

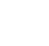


específicos de 'gobernanza' que deben encontrar su reflejo y medida en adecuados (y renovados) indicadores.

Estas acciones y estas estrategias persiguen la consecución de objetivos con repercusión económica y social: acumulación, sostenibilidad, productividad, competitividad.

En resumen, parece confirmarse la utilidad del concepto de espacio como concepto 'salvavidas' para avanzar en la comprensión de los procesos de producción, aplicación y transmisión de los conocimientos, en el caso concreto que nos ocupa de los conocimientos científicos y tecnológicos en relación, sobre todo, con procesos emergentes marcados por trayectorias evolutivas".

\subsection{Confluencia con nuevas aproximaciones a la economía del conocimiento y la innovación}

Por último, y de manera no menos importante, me parece oportuno traer a colación como ejemplo de trasplante compatible entre pensamientos de diferentes orígenes, la existencia de coincidencias entre los argumentos que he barajado en las revisiones diagnósticas sobre las relaciones entre conocimiento, innovación y socioeconomía con los argumentos que se desgranan a lo largo de las contribuciones del libro ya mencionado Economía del conocimiento y la innovación (Cañibano, Encinar y Muñoz, 2008).

Traigo aquí las partes del prólogo que ponen de manifiesto estas convergencias.

"Esta identificación de coincidencias se presenta como una muestra de existencia de procesos coevolutivos de convergencia intelectual, entre los que mencionaré los siguientes:

- Aplicación de importantes dosis de reflexión filosófica en una parte relevante de los textos integrantes del volumen.

- Aceptación de que el concepto de sistema se puede aplicar a cualquier nivel de organización desde un país hasta una empresa.

- Afirmación de la conexión entre la ciencia y las tecnologías genéricas.

- Establecimiento de la necesidad (conveniencia) de acercarse a los usuarios y a los productores para el desarrollo de tecnologías genéricas.

- Reconocimiento de la relevancia de la estrategia para el desarrollo de las innovaciones.

- Asunción del papel importante del capital social -en nuestro caso hemos tratado su influencia al analizar el concepto de espacio social de la biotecnología- (Muñoz y cols., 2006; también recogido en la sección titulada $L a$ biotecnología en el espejo, en la web www.institutoroche. es).

- Búsqueda del sentido de los indicadores tradicionales, discretos, de input y output, que en la visión predominante contemplan las actividades de I+D como cajas negras, sin que parezcan tener en cuenta la dinámica de los procesos que los hacen medibles.

- Pertinencia de pensar en la introducción de nuevas herramientas para analizar y promover las conexiones entre la producción y gestión del conocimiento con la innovación.

Después de este apartado de coincidencias, quisiera aprovechar el estímulo de las lecturas que nos ofrece el libro que editan Cañibano, Encinar y Muñoz para abrir alguna puerta a la disidencia, o mejor al debate. La primera puerta me lleva a atraer la atención al concepto de 'espacios' que he apuntado como alternativa al análisis y a las consiguientes interpretaciones del caso español en las políticas de I+D e innovación cuando se someten a un ejercicio comparativo con las políticas europeas sobre tales temas (Muñoz y cols., 2005, Muñoz, 2007). Me atrevo a proponer que las temáticas que se analizan y discuten en el libro Economía del conocimiento y la innovación. Nuevas aproximaciones... podrían tener cabida para su tratamiento analítico bajo la dimensión del término 'espacio tecnoeconómico', que sería una denominación adecuada a las dimensiones tratadas en el libro.

Una segunda cuestión que igualmente me atrevo a suscitar es la de empezar a pensar en la necesaria multidisciplinariedad que debería darse entre los responsables de la gestión de los programas de I+D. El énfasis de los discursos políticos en la innovación ha creado desconfianza, y un cierto sentimiento de marginación, en los científicos experimentales y en los ingenieros que fueron los tradicionales gestores del modelo de 'ciencia abierta' o de 'política para la ciencia', modelo que en buena parte se mantiene en los dos grandes países anglosajones, lideres de la generación de conocimiento, como Estados Unidos, Reino Unido -la figura de asesor del gobierno para los temas de ciencia y tecnología suele recaer en un científico experimental o en un ingeniero de reconocida excelencia profesional. Esta situación no se da en Europa donde el desarrollo de la políticas de ciencia y tecnología ha generado la incorporación de los científicos sociales a estas tareas, de modo particularmente relevante en el caso de Comisión Europea en la que se ha generado un maridaje entre estos expertos y la burocracia comunitaria 
que ha suscitado la sospecha de una parte muy relevante de la comunidad científica cultivadora de las ciencias específicamente experimentales.

Esta dinámica que no ha favorecido la conexión entre expertos de unos y otros campos científicos y académicos es además contradictoria, puesto que el énfasis de los programas I+D comunitarios ha estado puesto en los ámbitos científicos llamados 'duros' y en las tecnologías asociadas a ellos, con algunas modestas excepciones.

Dos datos son relevadores de que la situación es realmente conflictiva. Por un lado, la iniciativa de creación del European Research Council (ERC), promovida por la comunidad científica europea para rescatar la investigación europea de lo que esa comunidad considera control excesivo de los practicantes del discurso burocrático y económico. El segundo lo encontramos en el artículo de Dosi y colaboradores en esta misma obra en el que se vierten importantes críticas a la gestión de las políticas de I+D comunitarias y se realizan propuestas de rescate de los viejos valores de la ciencia abierta, propuestas que los propios autores avanzan aun a trueque de ser tildados de conservadores como ellos mismos reconocen.

Personalmente yo me alinearía en esta dirección y con idea de contribuir a la apertura del debate y la reflexión sobre este tema, expresaría mi agradecimiento a los economistas de la innovación que la asumen con tesis bien documentadas y reflejadas en este libro.

Estimo que si nos movemos por este 'espacio' -de nuevo recurro al término- se avanzaría mejor en la comprensión y la promoción de las conexiones entre conocimiento, su producción y gestión, con la innovación, a la par que se facilitaría la integración de los distintos actores y capacidades necesarias para que tales conexiones se lleven a cabo con eficacia y eficiencia".
Recibido: 19 de noviembre de 2008 Aceptado: 20 de enero de 2009

\section{BIBLIOGRAFÍA}

Cañibano Sánchez, Carolina; Encinar del Pozo, Maria Isabel y Muñoz Pérez, Félix-Fernando (coords.) (2008): Economía del conocimiento y la innovación. Nuevas aproximaciones a una relación compleja, Madrid, Ediciones Pirámide.

Consejo Superior de Investigaciones Científicas (2007): Tiempos de ciencia y de política. Homenaje a Emilio Muñoz, Madrid, CSIC.

Echeverría, Javier (2002): Ciencia y valores, Barcelona, Ediciones Destino (Colección Imago Mundial vol. 7).

Echeverría, Javier (2003): La revolución tecnocientífica, Madrid, Fondo de Cultura Económica de España.

Metcalfe, Stan (1995): "The economic foundations of technology policy: equilibrium and evolution perspectives", en P. Stoneman (ed.), Handbook of Economics of Innovation and Technology Change, pp. 409-512, 0xford, Blackwell.

Muñoz, Emilio (2002): "New socio-political environments and the dynamics of European public research systems",
Documento del trabajo CTS 02-20, http://www.iesam.csic.es/doctrab. htm.

Muñoz, Emilio (2005): "Gobernanza, ciencia tecnología y política: Trayectoria y evolución", en Gobernanza de la ciencia y la tecnología (Marta I. García y Oliver Todt, eds.), Arbor, vol. CLXXXI, n. ${ }^{\circ} 715$, pp. 287-300.

Muñoz, Emilio (2006): "Las humanidades y las ciencias sociales en el desarrollo regional y la innovación", en Las Ciencias Sociales y las Humanidades en los sistemas de Innovación (A. Ibarra, J. Castro y Liliana Rocca, eds.) pp. 115133, Bilbao, Servicio Editorial de la Universidad del País Vasco.

Muñoz, Emilio (2007): "Espacios de conocimientos y su gestión: procesos de Gobernanza", Revista lberoamericana de Ciencia, Tecnología y Sociedad, n. 8 , pp. 159-172.

Muñoz, Emilio (2008a): "Prólogo. Políticas científicas, innovación y conocimiento", en Economía del conocimiento y la innovación. Nuevas aproximaciones a una relación compleja (Cañibano, Ca- 
rolina; Encinar, Maria Isabel y Muñoz, Félix-Fernando, coords.), pp. 21-28, Madrid, Ediciones Pirámide.

Muñoz, Emilio (2008b): "La ciencia como motor de desarrollo y de cambio social. ¿Adónde nos conduce en el futuro?", Temas para el debate, n. ${ }^{\circ} 168$, noviembre, pp. 44-47.

Muñoz, Emilio y Ornia, Florencio (1986): Ciencia y Tecnología. Una oportunidad para España, Madrid, Ministerio de Educación y Ciencia, Aguilar S.A. de ediciones.

Muñoz, Emilio y García Arroyo, Arturo (2006): "El nacimiento de la Ley de la Ciencia: El sueño del progreso", mi+d revista electrónica, en papel número especial 20 años de la ley de la ciencia, diciembre 2006, pp. 21-25.

Muñoz, Emilio, Sebastián, Jesús (2007): "Exploración de la política científica en España: de la espeleología a la cartografia", en Cien años de política cientifica en España (Ana Romero de Pablos y M.a Jesús Santesmases, eds.), pp. 357-384, Madrid, Fundación BBVA.

Muñoz, Emilio; Plaza, Marta; Santos, David; Espinosa de los Monteros, Juan y Ponce, Gloria (2006): "El espacio social de la ciencia y la tecnología: percepción, comunicación y difusión", en Radiografía de la investigación pública en España (J. Sebastián y E. Muñoz, eds.), pp. 409-456, Madrid, Biblioteca Nueva.

Muñoz, Emilio; Santesmases, María J.; López Facal, Javier; Plaza, Luis M. y Todt, Oliver (2005): El espacio común de conocimiento en la Unión Europea. Un enfoque al problema desde España, Madrid, Academia Europea de Ciencias y Artes España.

Smits, Ruud y Kuhlmann, Stephan (2004): "The rise of systemic instruments in innovation", Int. Journal Foresight and Innovation Policy, vol. 1, n. ${ }^{\circ}$, 12, pp. 4-32. 